\title{
Harnack inequalities on a manifold with positive or negative Ricci curvature
}

\section{Dominique Bakry and Zhongmin M. Qian}

Summary. Several new Harnack estimates for positive solutions of the heat equation on a complete Riemannian manifold with Ricci curvature bounded below by a positive (or a negative) constant are established. These estimates are sharp both for small time, for large time and for large distance, and lead to new estimates for the heat kernel of a manifold with Ricci curvature bounded below.

\section{Introduction and main results.}

The main purpose of this paper is to present several new Harnack estimates for non-negative solutions of the heat equation on a complete manifold with Ricci curvature bounded below by a constant which may be positive or negative. To obtain Harnack inequalities, we first deduce gradient estimates, that is upper bounds of the gradient of the logarithm of a solution of the heat equation by a concave function of the time and the time derivative of the same quantity. Then, by standard methods, these bounds lead to Harnack inequalities and then to bounds on the heat kernel.

In this context, we obtain quite strong Harnack inequalities, which are improvements of the famous Li-Yau's estimate in [6], [12]. Although 
our methods are similar in both cases of positive and negative lower bound on the Ricci curvature, our results are completely new in the positive case, and are improvements of previous results in the negative one.

In order to state our results, we first introduce some basic notations: let $M$ be a complete Riemannian manifold of dimension $n$, and let $\Delta$ be the Laplace-Beltrami operator. Let $u$ be a positive solution of the heat equation

$$
\left(\Delta-\partial_{t}\right) u=0, \quad \text { on }[0, \infty) \times M
$$

and let $f=\log u$. Denote by $\nabla f$ the gradient of the function $f$ and by $f_{t}$ the time derivative of $f$.

In 1975, Yau [10] proved a Harnack inequality via Ricci curvature bounds for harmonic functions on a complete manifold. In their paper [6], Li and Yau have established a sharp Harnack inequality for parabolic harmonic functions on a complete manifold with non-negative Ricci curvature. Namely,

$$
|\nabla f|^{2}-f_{t} \leq \frac{n}{2 t}, \quad \text { for all } t>0
$$

They also proved the following gradient estimate for a manifold with Ricci curvature bounded below by $-K ; K \geq 0$

$$
|\nabla f|^{2}-\alpha f_{t} \leq \frac{n \alpha^{2}}{2 t}+\frac{n \alpha^{2} K}{2(\alpha-1)}, \quad \text { for all } t>0, \alpha>1
$$

In his book [6], Davies improved the previous inequality under the same assumption to the following one

(4) $|\nabla f|^{2}-\alpha f_{t} \leq \frac{n \alpha^{2}}{2 t}+\frac{n \alpha^{2} K}{4(\alpha-1)}, \quad$ for all $t>0, \alpha>1$.

Recently Yau [12] (also see Yau [11]) further established, among other things, the following gradient estimate: if Ric $\geq-K ; K \geq 0$, then

(5) $|\nabla f|^{2}-f_{t} \leq \sqrt{2 n K} \sqrt{|\nabla f|^{2}+\frac{n}{2 t}+2 n K}+\frac{n}{2 t}$, for all $t>0$.

With the method described in Section 4, it is standard to deduce from this a Harnack inequality close to (12) (see below), but with different 
constants. In Appendix A, with the method described in this paper, and under the same assumption, we will improve this inequality to

(6) $|\nabla f|^{2}-f_{t} \leq \sqrt{n K} \sqrt{|\nabla f|^{2}+\frac{n}{2 t}+\frac{n K}{4}}+\frac{n}{2 t}, \quad$ for all $t>0$,

which yields a Harnack inequality essentially similar to (12) for small time and large distance.

Let us also mention that Hamilton [8] has obtained a Harnack inequality for negative curvature manifolds.

The path to obtain Harnack inequalities is to first establish gradient estimates as (4) or (5). To begin with, let us state the main results of this paper. To this end, we first introduce two functions $X$ and $\widetilde{X}$ as follows: let $K \geq 0, n>0$ be two constants. Then the functions $X$ and $\widetilde{X}$ are defined on $(0, \infty) \times \mathbb{R}$ by

$$
X(t, Y)= \begin{cases}-\frac{n K}{2}+Y \\ +\sqrt{n K} \sqrt{\frac{n K}{4}-Y} \\ \cdot \operatorname{cotanh} \frac{2 t}{n} \sqrt{n K} \sqrt{\frac{n K}{4}-Y}, \quad Y \leq \frac{n K}{4} \\ -\frac{n K}{2}+Y \\ +\sqrt{n K} \sqrt{Y-\frac{n K}{4}} \\ \cdot \operatorname{cotan} \frac{2 t}{n} \sqrt{n K} \sqrt{Y-\frac{n K}{4}}, \quad Y>\frac{n K}{4} \\ \frac{n K}{2}+Y \quad, \quad Y<-\frac{n K}{4}, \\ +\sqrt{n K} \sqrt{Y+\frac{n K}{4}} \\ \cdot \operatorname{cotanh} \frac{2 t}{n} \sqrt{n K} \sqrt{Y+\frac{n K}{4}}, \quad Y \geq-\frac{n K}{4} \\ \frac{n K}{2}+Y \\ +\sqrt{n K} \sqrt{-Y-\frac{n K}{4}}\end{cases}
$$


respectively. Indeed, as we will see later, $X$ and $\tilde{X}$ are solutions of some simple differential equations. The key inequalities obtained in this paper are the following gradient estimates

Theorem 1. Suppose the Ricci curvature is bounded below by a constant $-K ; K \geq 0$, then we have:

1) $|\nabla f|^{2} \leq \tilde{X}\left(t, f_{t}\right)$, on $f_{t} \geq-n K / 4$.

2) For any $Y_{0} \geq-n K / 4$, we have

$$
|\nabla f|^{2} \leq \partial_{Y} \tilde{X}\left(t, Y_{0}\right)\left(f_{t}-Y_{0}\right)+\widetilde{X}\left(t, Y_{0}\right), \quad \text { for all } t>0 .
$$

3) There is a universal constant $c>0$, such that

$$
|\nabla f|^{2} \leq \tilde{X}\left(t, f_{t}\right), \quad \text { for all } 0<t \leq \frac{c}{K} .
$$

See Theorem 4 below for the precise value of the constant $c$. As a consequence, if Ric $\geq-K ; K \geq 0$, then

$$
-f_{t} \leq \frac{n}{2 t}+\frac{n K}{4}, \quad \text { for all } t>0
$$

which is very close to the best possible one could expect, since $(n-$ 1) $K / 4$ is the spectral gap of the space form with Ricci curvature $-K$. Indeed we will prove a better but slightly more complicated estimate than (9).

Then, by standard methods, we deduce from Theorem 1 the following Harnack inequality

$$
\frac{u(t, x)}{u(t+s, y)} \leq \exp \left(\frac{\rho^{2}}{4 s}+\int_{t}^{t+s}\left(C\left(\sigma, \frac{\rho^{2}}{4 s^{2}}\right)-A\left(\sigma, \frac{\rho^{2}}{4 s^{2}}\right)\right) d \sigma\right)
$$

for all $t \geq 0, s>0, x, y \in M$, where $\rho=d(x, y)$ is the geodesic distance from $x$ to $y$, and

$$
\begin{aligned}
C(t, Y)= & \tilde{X}(t, Y)-Y \\
= & \frac{n K}{2}+\sqrt{n K} \sqrt{Y+\frac{n K}{4}} \operatorname{cotanh} \frac{2 t}{n} \sqrt{n K} \sqrt{Y+\frac{n K}{4}} \\
& A(t, Y)=\frac{\partial_{Y} C(t, Y)\left(C(t, Y)-Y \partial_{Y} C(t, Y)\right)}{1+\partial_{Y} C(t, Y)}
\end{aligned}
$$


In particular, since $A(t, Y)>0$ when $Y \geq 0$, we have

$$
\begin{aligned}
\frac{u(t, x)}{u(t+s, y)} \leq & \left(\frac{\sinh \left(\frac{2}{n}(t+s) \sqrt{n K} \sqrt{\frac{\rho^{2}}{4 s^{2}}+\frac{n K}{4}}\right)}{\sinh \left(\frac{2}{n} t \sqrt{n K} \sqrt{\frac{\rho^{2}}{4 s^{2}}+\frac{n K}{4}}\right)}\right)^{n / 2} \\
& \cdot \exp \left(\frac{\rho^{2}}{4 s}+\frac{n K}{2} s\right) .
\end{aligned}
$$

By an elementary computation, inequality (10) yields the following

$$
\begin{aligned}
\frac{u(t, x)}{u(t+s, y)} \leq & \left(\frac{t+s}{t}\right)^{n / 2} \\
& \cdot \exp \left(\frac{(\rho+\sqrt{K n} s)^{2}}{4 s}+\frac{\sqrt{n K}}{4} \min \{\rho, \sqrt{n K} s\}\right),
\end{aligned}
$$

for all $s>0, x, y \in M$. We will give an independent proof of this in Section 4. We have been informed by Professor S. T. Yau that he already obtained a Harnack inequality in this context, see [15], [14].

As usual, Harnack inequalities lead to lower bounds of the heat kernel. Let $H(t, x, y)$ be the heat kernel: the fundamental solution of the heat equation (1). Then (12) implies that

$$
\begin{aligned}
H(t, x, y) \geq & \frac{1}{(4 \pi t)^{n / 2}} \\
& \cdot \exp \left(-\frac{(\rho+\sqrt{K n} t)^{2}}{4 t}-\frac{\sqrt{n K}}{4} \min \{\rho, \sqrt{n K} t\}\right),
\end{aligned}
$$

for all $(t, x, y) \in(0, \infty) \times M \times M, \rho=d(x, y)$. See [3], [8] for a comparison theorem for heat kernels.

Notice that the leading term in (13) for small time is

$$
\frac{1}{(4 \pi t)^{n / 2}} \exp \left(-\frac{\rho^{2}}{4 t}\right)
$$

for large time is

$$
\exp \left(-\frac{n K}{4} t\right)
$$

for large distance is

$$
\exp \left(-\frac{\rho^{2}}{4 t}-\frac{\sqrt{n K}}{2} \rho\right)
$$


They are all very close to those best possibles we could expect.

For positive Ricci curvature manifolds, we will prove the following gradient estimate

Theorem 2. Let Ric $\geq K ; K \geq 0$. Then

1) $|\nabla f|^{2} \leq X\left(t, f_{t}\right)$, on $f_{t} \leq n K / 4$.

2) If $t \geq 2 / K$, then $|\nabla f|^{2} \leq X\left(t, f_{t}\right)$ and $-n /(2 t) \leq f_{t} \leq n K / 4$.

3) If $t \leq 2 / K$, then

$$
|\nabla f|^{2} \leq X\left(t, f_{t}\right), \quad \text { on } f_{t} \leq Y_{0}
$$

and

$$
|\nabla f|^{2} \leq \partial_{Y} X\left(t, Y_{0}\right)\left(f_{t}-Y_{0}\right)+X\left(t, Y_{0}\right), \quad \text { on } f_{t}>Y_{0}
$$

where

$$
Y_{0}=\left(1+\frac{\pi^{2}}{64}\right) \frac{n K}{4}
$$

It turns out that both $|\nabla f|^{2}$ and $f_{t}$ are uniformally bounded for each $t \geq 2 / K$ if Ric $\geq K>0$.

We could also deduce from this a Harnack inequality, but it takes a more complicated form than in the negative curvature case, and we will therefore omit it in this paper.

The main tool used in this paper is the maximum principle, which plays a fundamental rôle in Partial Differential Equations theory, see for example [5]. Although the basic idea adopted in this paper is to apply the maximum principle and Bochner identity to some nice test functions; this has been developed in a series of papers by Yau [9], [10], [12], Cheng and Yau [4], Li and Yau [6] etc. (see [7] for more references); the main difficulty with this method relies on the fact that, for any family of test functions, one gets different kind of results, and therefore the test functions in use are related to the results one is looking for. But it is not always easy (and indeed quite hard in general) to device what is the best estimate one could expect from a given differential inequality. Our main contribution in this context is to develop a method which produces the best possible estimates and to show how to construct good test functions in order to prove the expected estimates via the maximum principle. This method applies to a more general setting 
than the one described here, and it could be used in different contexts for more general equations.

Let us explain our main idea as following. Everything relies on the three following equations satisfied by $|\nabla f|^{2}$ and $f_{t}$

$$
\begin{gathered}
\Delta f=f_{t}-|\nabla f|^{2}, \\
\left(\widetilde{\Delta}-\partial_{t}\right) f_{t}=0
\end{gathered}
$$

and

$$
\left(\widetilde{\Delta}-\partial_{t}\right)|\nabla f|^{2}=2|\operatorname{Hess} f|^{2}+2 \operatorname{Ric}(\nabla f, \nabla f),
$$

where $\widetilde{\Delta}=\Delta+2 \nabla f$ which is an elliptic operator. See Section 2 for detail.

(16) comes from the Bochner identity. Therefore, if $K$ is a lower bound of the Ricci curvature, then we have the following inequality

$$
\left(\widetilde{\Delta}-\partial_{t}\right)|\nabla f|^{2} \geq \frac{2}{n}(\Delta f)^{2}+2 K|\nabla f|^{2} .
$$

Inserting (14) into (17), we end up two differential inequalities

$$
\begin{aligned}
& \left(\widetilde{\Delta}-\partial_{t}\right)|\nabla f|^{2} \geq \frac{2}{n}\left(|\nabla f|^{2}-f_{t}\right)^{2}+2 K|\nabla f|^{2}, \\
& \left(\widetilde{\Delta}-\partial_{t}\right) f_{t}=0 .
\end{aligned}
$$

The main point of this paper is to compare $\left(|\nabla f|^{2}, f_{t}\right)$ with the solution $(X, Y)$ of the following system of differential equations

$$
\begin{aligned}
& -\partial_{t} X=\frac{2}{n}(X-Y)^{2}+2 K X, \\
& -\partial_{t} Y=0,
\end{aligned}
$$

with the condition that $X(0)=\infty$. Since $Y=$ constant, we regard it as a parameter, and write the solution as $X=X(t, Y)$. It is easy to see that if $K \geq 0$, then $X(t, Y)$ is the function defined by (7), and if $K \leq 0$, then $X(t, Y)=\tilde{X}(t, Y)$ with $-K$ in (8).

In fact, we were not able to prove (and we do not think it is true) that $|\nabla f|^{2} \leq X\left(t, f_{t}\right)$ everywhere, and this comes from the lack of concavity of the curve $Y \longrightarrow X(t, Y)$. What we show is that this inequality holds on the most part of the curve. More precisely, our 
main result asserts that if $\mathrm{Ric} \geq K$, then the most part of the curve $\left(X\left(t, f_{t}\right), f_{t}\right)$ is above the curve $\left(|\nabla f|^{2}, f_{t}\right)$. In other words, $|\nabla f|^{2} \leq$ $X\left(t, f_{t}\right)$ for most of the values of $f_{t}$, and we have a linear upper bound on the remaining part.

The paper is organised as follows. In Section 2 we establish gradient estimates and some consequences for manifolds with Ricci curvature bounded below. Section 3 deals with the case of positive Ricci curvature manifolds. We deduce Harnack inequalities in Section 4. In Section 5, we describe several extensions to other diffusion operators, and, in the end, we give an improved form of Yau's gradient estimate.

The results obtained in this paper have been announced in [2].

\section{Gradient estimates for complete manifolds.}

The main purpose of this section is to prove Theorem 1. Thus throughout this section it will be assumed that Ric $\geq-K$, where $K \geq 0$ is a constant.

Let $u$ be a positive solution of the heat equation

$$
\left(\Delta-\partial_{t}\right) u=0, \quad \text { on }[0, \infty) \times M
$$

and let $f=\log u$. One can easily see that

$$
\left(\Delta-\partial_{t}\right) f=-\Gamma(f, f),
$$

where $\Gamma(f, f)=|\nabla f|^{2}$. In general, if $\Delta$ is replaced by any sub-elliptic differential operator, we may define

$$
\Gamma(g, h)=\frac{1}{2}(\Delta(h g)-h \Delta g-g \Delta h), \quad \text { for all } g, h \in C^{\infty}(M),
$$

and therefore $\Gamma(g, h)$ will stand for $\langle\nabla g, \nabla h\rangle$.

Differentiating (21) in $t$, we obtain the first fundamental equation

$$
\Delta f_{t}+2\left\langle\nabla f, \nabla f_{t}\right\rangle-\partial_{t} f_{t}=0
$$

Then, define the bilinear operator $\Gamma_{2}$ by iterating the previous definition of $\Gamma$

$$
\Gamma_{2}(g, h)=\frac{1}{2}(\Delta \Gamma(g, h)-\Gamma(g, \Delta h)-\Gamma(\Delta g, h)),
$$


for all $g, h \in C^{\infty}(M)$. Using $\Gamma_{2}$, we may rewrite the classical Bochner identity as

$$
\Gamma_{2}(g, h)=\langle\operatorname{Hess} g, \operatorname{Hess} h\rangle+\operatorname{Ric}(\nabla g, \nabla h),
$$

for all $g, h \in C^{\infty}(M)$. Since Ric $\geq-K$ and $|\operatorname{Hess} g| \geq(\Delta g)^{2} / n$, Bochner identity yields the following curvature-dimension inequality

$$
\Gamma_{2}(g, g) \geq \frac{1}{n}(\Delta g)^{2}-K \Gamma(g, g), \quad \text { for all } g \in C^{\infty}(M) .
$$

This is the only form in which the Ricci curvature will appear in what follows. Then, the fundamental remark is that, using (21) and (23) and the previous definition of $\Gamma_{2}$, we get another fundamental equation

$$
\Delta \Gamma(f, f)+2\langle\nabla f, \nabla \Gamma(f, f)\rangle-\partial_{t} \Gamma(f, f)=2 \Gamma_{2}(f, f) .
$$

For simplicity, introduce a differential operator: $L=\Delta+2 \nabla f-\partial_{t}$. Then the basic equations (22) and (26) can be rewritten

$$
L|\nabla f|^{2}=2 \Gamma_{2}(f, f), \quad L f_{t}=0 .
$$

If we notice that (21) can be rewritten as

$$
-\Delta f=|\nabla f|^{2}-f_{t}
$$

then the curvature-dimension inequality, implies that

$$
L|\nabla f|^{2} \geq \frac{2}{n}\left(|\nabla f|^{2}-f_{t}\right)^{2}-2 K|\nabla f|^{2} .
$$

We next look for a smooth function $B$ on $(0, \infty) \times \mathbb{R}$ such that

$$
|\nabla f|^{2}-f_{t} \leq B\left(t, f_{t}\right), \quad \text { for all } t>0 \text {. }
$$

To this end, we set $F=|\nabla f|^{2}-f_{t}-B\left(t, f_{t}\right)$, and $G=t F$. By the fact that $L f_{t}=0$, we have

$$
L B\left(t, f_{t}\right)=-\partial_{t} B\left(t, f_{t}\right)+\partial_{Y}^{2} B\left(t, f_{t}\right)\left|\nabla f_{t}\right|^{2} .
$$

Therefore

$$
\begin{aligned}
L F & =L|\nabla f|^{2}-L B\left(t, f_{t}\right) \\
& =L|\nabla f|^{2}-\partial_{Y}^{2} B\left(t, f_{t}\right)\left|\nabla f_{t}\right|^{2}+\partial_{t} B\left(t, f_{t}\right) \\
& =2 \Gamma_{2}(f, f)-\partial_{Y}^{2} B\left(t, f_{t}\right)\left|\nabla f_{t}\right|^{2}+\partial_{t} B\left(t, f_{t}\right) .
\end{aligned}
$$


Thus if $\partial_{Y}^{2} B \leq 0$ (which means $B$ is concave in $Y$ ), then

$$
\begin{aligned}
L F & \geq \frac{2}{n}\left(|\nabla f|^{2}-f_{t}\right)^{2}-2 K|\nabla f|^{2}+\partial_{t} B\left(t, f_{t}\right) \\
& =\frac{2}{n}(F+B)^{2}-2 K|\nabla f|^{2}+\partial_{t} B\left(t, f_{t}\right) \\
& =\frac{2}{n} F^{2}+\frac{4}{n} B F+\frac{2}{n} B^{2}-2 K|\nabla f|^{2}+\partial_{t} B\left(t, f_{t}\right) \\
& =\frac{2}{n} F^{2}+\left(\frac{4}{n} B-2 K\right) F+\partial_{t} B+\frac{2}{n} B^{2}-2 K\left(f_{t}+B\right) .
\end{aligned}
$$

Hence

$$
\begin{aligned}
L G= & -F+t L F \\
\geq & \frac{2 t}{n} F^{2}+\left(\frac{4 t}{n} B-2 K t-1\right) F \\
& -2 K t\left(f_{t}+B\right)+\frac{2 t}{n} B^{2}+t \partial_{t} B\left(t, f_{t}\right) .
\end{aligned}
$$

Next we specify the function $B$, so that

$$
|\nabla f|^{2}-f_{t} \leq B\left(t, f_{t}\right), \quad \text { for all } t>0 .
$$

More precisely, for any $Y_{0}>-n K / 4$, we shall produce a function $B$ depending on the parameter $Y_{0}$ for which we shall prove the above upper bound.

To this end, consider the solution $C$ of the differential equation on the half line $(0, \infty)$ with a parameter $Y \in \mathbb{R}$

$$
\partial_{t} C+\frac{2}{n} C^{2}-2 K(Y+C)=0, \quad C(0)=\infty .
$$

Then if $Y>-n K / 4$, we find that

$$
C(t, Y)=\tilde{X}(t, Y)-Y=\frac{n K}{2}+\frac{n}{2 t} \frac{b(t, Y)}{2} \operatorname{cotanh} \frac{b(t, Y)}{2}
$$

where

$$
b(t, Y)=\frac{4 t}{n} \sqrt{n K} \sqrt{Y+\frac{n K}{4}} .
$$


It is easily seen that

$$
\partial_{Y} b(t, Y)=\left(\frac{4 t}{n}\right)^{2} \frac{n K}{2} \frac{1}{b(t, Y)}
$$

so that

$$
\begin{aligned}
\partial_{Y} C & =\frac{n}{4 t}\left(\partial_{Y} b+\frac{2 \partial_{Y} b}{e^{b}-1}-\frac{2 b \partial_{Y} b e^{b}}{\left(e^{b}-1\right)^{2}}\right) \\
& =2 K t\left(\frac{1}{b}+\frac{2}{b\left(e^{b}-1\right)}-\frac{2 e^{b}}{\left(e^{b}-1\right)^{2}}\right) .
\end{aligned}
$$

Therefore

$$
\lim _{Y \rightarrow-n K / 4} C(t, Y)=\frac{n}{2 t}+\frac{n K}{2}
$$

and

$$
\lim _{Y \rightarrow-n K / 4} \partial_{Y} C(t, Y)=\frac{2 K}{3} t, \quad \lim _{b \rightarrow \infty} \partial_{Y} C(t, Y)=0 .
$$

Moreover, for each $t>0$, the function $Y \longrightarrow C(t, Y)$ is concave on the interval $(-n K / 4, \infty)$.

Taking derivative with respect to $Y$ in (34) we get that

$$
\partial_{t} \partial_{Y} C+\frac{4}{n} C \partial_{Y} C-2 K\left(1+\partial_{Y} C\right)=0 .
$$

Let $Y_{0}>-n K / 4$, and take $B$ to be the linearization of $C$ at $Y_{0}$, i.e.

$$
B(t, Y)=\partial_{Y} C\left(t, Y_{0}\right)\left(Y-Y_{0}\right)+C\left(t, Y_{0}\right) .
$$

Then

$$
\partial_{t} B=\partial_{t} \partial_{Y} C\left(t, Y_{0}\right)\left(Y-Y_{0}\right)+\partial_{t} C\left(t, Y_{0}\right)
$$

and

$$
\begin{aligned}
& \frac{2}{n} B^{2}= \frac{2}{n}\left(\partial_{Y} C\left(t, Y_{0}\right)\left(Y-Y_{0}\right)+C\left(t, Y_{0}\right)\right)^{2} \\
&= \frac{2}{n}\left(\partial_{Y} C\left(t, Y_{0}\right)\left(Y-Y_{0}\right)\right)^{2}+\frac{4}{n} C\left(t, Y_{0}\right) \partial_{Y} C\left(t, Y_{0}\right)\left(Y-Y_{0}\right) \\
&+\frac{2}{n} C\left(t, Y_{0}\right)^{2}, \\
& 2 K(Y+B)=2 K\left(Y_{0}+C\left(t, Y_{0}\right)\right)+2 K\left(Y-Y_{0}\right)\left(1+\partial_{Y} C\left(t, Y_{0}\right)\right) .
\end{aligned}
$$


Therefore, by (34) and (40), we get that

$$
\partial_{t} B+\frac{2}{n} B^{2}-2 K(Y+B)=\frac{2}{n}\left(\partial_{Y} C\left(t, Y_{0}\right)\left(Y-Y_{0}\right)\right)^{2} .
$$

Now define $F=|\nabla f|^{2}-f_{t}-B\left(t, f_{t}\right)$, where the constant $Y_{0}>-n K / 4$ in the definition of the function $B$. Let $G=t F$. Then by (33), we have

$$
\begin{aligned}
L G \geq & \frac{2}{n t} G^{2}+\left(\frac{4 B}{n}-2 K-\frac{1}{t}\right) G+t\left(\partial_{t} B+\frac{2}{n} B^{2}-2 K\left(f_{t}+B\right)\right) \\
= & \frac{2}{n t} G^{2}+\left(\frac{4}{n} \partial_{Y} C\left(t, Y_{0}\right)\left(f_{t}-Y_{0}\right)\right) G+\left(\frac{4}{n} C\left(t, Y_{0}\right)-2 K-\frac{1}{t}\right) G \\
(43) & +\frac{2 t}{n}\left(\partial_{Y} C\left(t, Y_{0}\right)\left(f_{t}-Y_{0}\right)\right)^{2} \\
= & \frac{2}{n t}\left(G+t \partial_{Y} C\left(t, Y_{0}\right)\left(f_{t}-Y_{0}\right)\right)^{2}+\left(\frac{4}{n} C\left(t, Y_{0}\right)-2 K-\frac{1}{t}\right) G .
\end{aligned}
$$

However,

$$
\frac{4 t}{n} C\left(t, Y_{0}\right)-2 K t=b\left(t, Y_{0}\right)+\frac{2 b\left(t, Y_{0}\right)}{e^{b\left(t, Y_{0}\right)}-1},
$$

and therefore by the elementary inequality

$$
b+\frac{2 b}{e^{b}-1} \geq 2, \quad \text { for all } b>0,
$$

we have

$$
\frac{4}{n} C\left(t, Y_{0}\right)-2 K-\frac{1}{t} \geq \frac{1}{t}, \quad \text { for all } t>0, Y_{0} \geq-\frac{n K}{4} .
$$

Hence by (43)

$$
L G \geq \frac{2}{n t}\left(G+t \partial_{Y} C\left(t, Y_{0}\right)\left(f_{t}-Y_{0}\right)\right)^{2}+\frac{1}{t} G .
$$

If the manifold is compact, consider a point $\left(t_{0}, x\right)$ at which the maximum of $G$ on $[0, t] \times M$ is attained: then, at this point, by the maximum principle, $\widetilde{\Delta}(G) \leq 0$. Moreover, $\partial G / \partial t \geq 0$ and $\nabla G=0$. From these we conclude that $G \leq 0$. In this case we have

$$
|\nabla f|^{2}-f_{t} \leq \partial_{Y} C\left(t, Y_{0}\right)\left(f_{t}-Y_{0}\right)+C\left(t, Y_{0}\right)
$$


In the case where the manifold $M$ is non-compact, we will get the same conclusion with a slight modification in the arguments. Since Ricci curvature is bounded below, we may use a generalised maximum principle (see [12], [5]) as following: replace $K$ by any $\widetilde{K}>K$ in the definition of the function $C$. Then the same argument yields the following inequality

$$
\begin{aligned}
L G & \geq \frac{2}{n t}\left(G+t \partial_{Y} C\left(t, Y_{0}\right)\right)^{2}+\frac{1}{t} G+2 t(\widetilde{K}-K)|\nabla f|^{2} \\
& \geq \frac{1}{t} G+2 t(\widetilde{K}-K)|\nabla f|^{2} .
\end{aligned}
$$

Using then the Li-Yau's estimate (3), we may check that

$$
G(t, \cdot) \leq \frac{n}{2}\left(1+\partial_{Y} C\left(t, Y_{0}\right)\right)^{2}+\frac{n K}{4}\left(1+\partial_{Y} C\left(t, Y_{0}\right)\right)^{2} \frac{t}{\partial_{Y} C\left(t, Y_{0}\right)} .
$$

However,

$$
\lim _{t \rightarrow 0} \frac{t}{\partial_{Y} C\left(t, Y_{0}\right)}=\frac{3}{2 \widetilde{K}} .
$$

Therefore for any $t>0$,

$$
\sup _{[0, t] \times M} G<\infty .
$$

Thus we can use the generalised maximum principle to the function $G$ on $[0, t] \times M$ for any fixed $t>0$ : if $\sup _{[0, t] \times M} G>0$, then we may find a point $t_{0} \in[0, t]$ and a sequence of points $\left\{x_{k}\right\} \in M$, such that

$$
\Delta G\left(t_{0}, x_{k}\right) \leq \frac{1}{k}, \quad|\nabla G|\left(t_{0}, x_{k}\right) \leq \frac{1}{k} .
$$

$G(0, \cdot) \leq 0$, and therefore $t_{0}>0$. Also,

$$
\partial_{t} G\left(t_{0}, x_{k}\right) \geq 0, \quad \lim _{k \rightarrow \infty} G\left(t_{0}, x_{k}\right)=\sup _{[0, t] \times M} G .
$$

Hence we have

$$
L G\left(t_{0}, x_{k}\right) \leq \frac{1}{k}+\frac{2}{k}|\nabla f|,
$$

which together with (46) implies that

$$
\begin{aligned}
\frac{1}{k} & \geq L G\left(t_{0}, x_{k}\right)-\frac{2}{k}|\nabla f| \\
& \geq \frac{1}{t_{0}} G\left(x_{k}\right)+2 t_{0}(\widetilde{K}-K)|\nabla f|^{2}-\frac{2}{k}|\nabla f| \\
& \geq \frac{1}{t_{0}} G\left(x_{k}\right)-\frac{1}{2(\widetilde{K}-K) t_{0}} \frac{1}{k^{2}} .
\end{aligned}
$$


Letting $k \longrightarrow \infty$ we get that

$$
0 \geq \frac{1}{t_{0}} \sup _{[0, t] \times M} G
$$

which is a contradiction to the assumption that $\sup _{[0, t] \times M} G>0$. Therefore $G \leq 0$. Since $\widetilde{K}>K$ is arbitrary, we have proved the main result of this section:

Theorem 3. Let Ric $\geq-K ; K \geq 0$, and let $f=\log u$, where $u$ is a positive solution of the heat equation. Then

$$
\begin{aligned}
|\nabla f|^{2} & \leq \widetilde{X}\left(t, f_{t}\right), \quad \text { on } f_{t}>-\frac{n K}{4}, \\
|\nabla f|^{2}-f_{t} & \leq \frac{2 K}{3} t\left(f_{t}+\frac{n K}{4}\right)+\frac{n}{2 t}+\frac{n K}{2} \\
& \leq \frac{n}{2 t}+\frac{n K}{2}, \quad \text { on } f_{t} \leq-\frac{n K}{4}
\end{aligned}
$$

and

$$
|\nabla f|^{2}-f_{t} \leq \inf _{Y_{0}>-n K / 4}\left(\partial_{Y} C\left(t, Y_{0}\right)\left(f_{t}-Y_{0}\right)+C\left(t, Y_{0}\right)\right)
$$

Proof. We have proved (49). By taking $Y_{0}=f_{t}$ and noticing that $\widetilde{X}(t, Y)=Y+C(t, Y)$ we get (47). Letting $Y_{0} \longrightarrow-n K / 4$ we get (48). So we completed the proof.

REMARK. In the above proof, we in fact used a "parabolic version" of Yau's generalised maximum principle. Indeed, if we apply Yau's argument in [12] to the product manifold $[0, t] \times M$ (with boundary), and use the Hopf's maximum principle by Hopf (i.e. maximum principle with boundaries), since $[0, t]$ is compact, we can easily obtain the parabolic version of the generalised maximum principle.

Corollary 1. Let Ric $\geq-K, K \geq 0$, and let $f=\log u$, where $u$ is $a$ positive solution of the heat equation. Then we have

$$
-f_{t} \leq \frac{1}{1+\frac{2}{3} K t}\left(\frac{n}{2 t}+\frac{n K}{4}\right)+\frac{n K}{4} \leq \frac{n}{2 t}+\frac{n K}{4} .
$$


Proof. For any $Y_{0}>-n K / 4$, we have

$$
-f_{t} \leq \frac{C\left(t, Y_{0}\right)-Y_{0} \partial_{Y} C\left(t, Y_{0}\right)}{1+\partial_{Y} C\left(t, Y_{0}\right)}
$$

By letting $Y_{0} \longrightarrow-n K / 4$, we get the conclusion.

We note that

$$
\frac{n}{2 t} \leq \frac{1}{1+\frac{2}{3} K t}\left(\frac{n}{2 t}+\frac{n K}{4}\right)+\frac{n K}{4},
$$

that is, the upper bound of $-f_{t}$ would not be better than $n /(2 t)$ for negative curvature manifolds. However one would expect that the best upper bound of $-f_{t}$ should be $n /(2 t)+(n-1) K / 4$, as $(n-1) K / 4$ is the spectral gap of the heat semi-group of the constant curvature space form with Ricci curvature $-K$. But we can see that

$$
\frac{n}{2 t}+\frac{(n-1) K}{4} \leq \frac{1}{1+\frac{2}{3} K t}\left(\frac{n}{2 t}+\frac{n K}{4}\right)+\frac{n K}{4}
$$

if and only if $t \geq(n-3) /(2 K)$. Therefore, if the dimension of $M$ is bigger than 3, then our upper bound is even better than the expected one: $n /(2 t)+(n-1) K / 4$, within the time range $(0,(n-3) /(2 K))$.

Corollary 2. Let Ric $\geq-K ; K \geq 0$, and let $H(t, x, y)$ be the heat kernel. Then

$$
H(t, x, x) \geq \frac{1}{(4 \pi t)^{n / 2}}\left(1+\frac{2}{3} K t\right)^{n / 8} e^{-n K t / 4},
$$

for all $t>0, x \in M$.

Proof. By Corollary 1, we have

$$
\begin{aligned}
-\partial_{t} \log (4 \pi t)^{n / 2} H & =-\partial_{t} \log H-\frac{n}{2 t} \\
& \leq \frac{1}{1+\frac{2}{3} K t}\left(\frac{n}{2 t}+\frac{n K}{4}\right)-\frac{n}{2 t}+\frac{n K}{4} \\
& =-\frac{n K}{4} \frac{1}{(3+2 K t)}+\frac{n K}{4} .
\end{aligned}
$$


Using the fact that

$$
\lim _{t \rightarrow 0}(4 \pi t)^{n / 2} H(t, x, x)=1
$$

and integrating both sides over $[0, t]$, we get the conclusion.

Corollary 3. Let Ric $\geq-K ; K \geq 0$. Then

$$
|\nabla f|^{2}-f_{t} \leq \sqrt{n K} \sqrt{f_{t}+\frac{n K}{4}}+\frac{n}{2 t}+\frac{n K}{2}, \quad \text { on } f_{t} \geq-\frac{n K}{4}
$$

and

$$
|\nabla f|^{2}-f_{t} \leq \frac{2 K}{3} t\left(f_{t}+\frac{n K}{4}\right)+\frac{n}{2 t}+\frac{n K}{2}
$$

Proof. We only need to prove the first inequality. Since

$$
\begin{aligned}
\tilde{X}(t, Y) & \leq Y+\frac{n K}{2}+\frac{n}{2 t}\left(1+\frac{b}{2}\right) \\
& =Y+\frac{n}{2 t}+\frac{n K}{2}+\sqrt{n K} \sqrt{Y+\frac{n K}{4}},
\end{aligned}
$$

(54) follows immediately from Theorem 3.

By estimate (51), we have

$$
-f_{t} \leq \frac{n}{2 t}+\frac{n K}{4}
$$

With this estimate, we can prove a better gradient estimate.

Indeed, let $C$ be the solution of the differential equation (34) on the half line $(0, \infty)$ with a parameter $Y<-n K / 4$ and $C(0)=\infty$. Then

$$
C(t, Y)=\frac{n K}{2}+\frac{n}{2 t} \frac{b(t, Y)}{2} \operatorname{cotan} \frac{b(t, Y)}{2}
$$

with

$$
b(t, Y)=\frac{4 t}{n} \sqrt{n K} \sqrt{-Y-\frac{n K}{4}} .
$$


Note that the function $C$, defined by (35) for $Y>-n K / 4$ and by (55) for $Y<-n K / 4$ and

$$
C\left(t,-\frac{n K}{4}\right)=\frac{n K}{2}+\frac{n}{2 t}
$$

is a smooth function on $(0, \infty) \times \mathbb{R}$. However, $Y \longrightarrow C(t, Y)$ is not concave on $(-\infty,-n K / 4)$.

It is easy to see that $\widetilde{X}(t, Y)=Y+C(t, Y)$ for all $Y \in \mathbb{R}$.

Up to now, we restricted our attention to the part $Y \geq-n K / 4$ of the curve $Y \longrightarrow C(t, Y)$. In what follows, we are going to improve the previous estimate for any value of $Y$ provided that the time $t$ is not too big.

Let $c_{K}$ be the positive constant

$$
\frac{\pi^{2}}{32} \frac{1}{K}
$$

Then, we have

Theorem 4. Let Ric $\geq-K ; K \geq 0$, and let $f=\log u$, where $u$ is $a$ positive solution of the heat equation. Then for any $0<t \leq c_{K}$,

$$
|\nabla f|^{2} \leq \tilde{X}\left(t, f_{t}\right)
$$

In fact, fix any $0<t \leq c_{K}$, and let $s \in(0, t]$. Let

$$
Y_{0} \in\left[-\frac{n}{2 t}-\frac{n K}{4},-\frac{n K}{4}\right)
$$

and let

$$
B(s, Y)=\partial_{Y} C\left(s, Y_{0}\right)\left(Y-Y_{0}\right)+C\left(s, Y_{0}\right) .
$$

Define a test function as usual: $F=|\nabla f|^{2}-f_{s}-B\left(s, f_{s}\right)$ and $G=s F$. Then the same argument as above yields that

$$
L G \geq \frac{2}{n s}\left(G+s \partial_{Y} C\left(s, Y_{0}\right)\left(f_{s}-Y_{0}\right)\right)^{2}+\left(\frac{4}{n} C\left(s, Y_{0}\right)-2 K-\frac{1}{s}\right) G
$$

Notice that

$$
\frac{4 s}{n} C\left(s, Y_{0}\right)-2 K s=b\left(s, Y_{0}\right) \operatorname{cotan} \frac{b\left(s, Y_{0}\right)}{2},
$$


as $Y_{0}<-n K / 4$, where

$$
\frac{b\left(s, Y_{0}\right)}{2}=\frac{2 s}{n} \sqrt{n K} \sqrt{-Y_{0}-\frac{n K}{4}} .
$$

Since $s \leq t \leq c_{K}$, we have

$$
\frac{b\left(s, Y_{0}\right)}{2} \leq \frac{2 s}{n} \sqrt{n K} \sqrt{\frac{n}{2 t}} \leq \frac{2 t}{n} \sqrt{n K} \sqrt{\frac{n}{2 t}} \leq \frac{\pi}{4} .
$$

Therefore, for any $s \leq t \leq c_{K}$, and

$$
Y_{0} \in\left[-\frac{n}{2 t}-\frac{n K}{4},-\frac{n K}{4}\right),
$$

we have

$$
b\left(s, Y_{0}\right) \operatorname{cotan} \frac{b\left(s, Y_{0}\right)}{2} \geq 2 \cos \frac{b\left(s, Y_{0}\right)}{2} \geq \sqrt{2} .
$$

Hence, for those $s$ and $Y_{0}$, we have

$$
L G \geq \frac{2}{n s}\left(G+s \partial_{Y} C\left(s, Y_{0}\right)\left(f_{s}-Y_{0}\right)\right)^{2}+\frac{\sqrt{2}-1}{s} G,
$$

and by applying the maximum principle to $G$ on $[0, t] \times M$, we conclude that

$$
|\nabla f|^{2}-f_{s} \leq \partial_{Y} C\left(s, Y_{0}\right)\left(f_{s}-Y_{0}\right)+C\left(s, Y_{0}\right)
$$

for any $0<s \leq t \leq c_{K}$ and

$$
Y_{0} \in\left[-\frac{n}{2 t}-\frac{n K}{4},-\frac{n K}{4}\right) .
$$

In particular if $f_{t} \leq-n K / 4, t \leq c_{K}$, since $f_{t}>-n /(2 t)-n K / 4$, we can take $Y_{0}=f_{t}$ in (58) to get that

$$
|\nabla f|^{2}-f_{t} \leq C\left(t, f_{t}\right) .
$$

Thus we completed the proof.

By the above proof, we also proved in fact the following 
Theorem 5. Let Ric $\geq-K ; K \geq 0$, and let $f=\log u$, where $u$ is a positive solution of the heat equation. Then for any $Y_{0} \leq-n K / 4$,

$$
|\nabla f|^{2}-f_{t} \leq \partial_{Y} C\left(t, Y_{0}\right)\left(f_{t}-Y_{0}\right)+C\left(t, Y_{0}\right),
$$

for any

$$
t \leq \frac{\pi n}{8 \sqrt{n K} \sqrt{-Y_{0}-\frac{n K}{4}}} .
$$

Corollary 4. Let Ric $\geq-K ; K \geq 0$, and let $f=\log u$, where $u$ is a positive solution of the heat equation. Then

1) If $0<t \leq c_{K}$, then $f_{t} \geq Y_{1}(t)$, where $Y_{1}(t)$ is the unique solution of the equation

$$
Y+C(t, Y)=0, \quad Y<0 .
$$

2) For any $t>0$,

$$
-f_{t} \leq \frac{C\left(t, Z_{t}\right)-Z_{t} \partial_{Y} C\left(t, Z_{t}\right)}{1+\partial_{Y} C\left(t, Z_{t}\right)}
$$

where

$$
-Z_{t}=\frac{n K}{4}+\frac{\pi^{2}}{64} \frac{n}{K t^{2}}
$$

\section{Positive curvature manifold.}

The goal of this section is to prove Theorem 2. The method follows exactly the same lines as in the previous section, although the conclusions are quite different.

Let $M$ be a Riemannian manifold with dimension $n$, such that Ric $\geq K$, where $K$ is a positive constant.

Let $f=\log u$, and $u$ be a positive solution of the heat equation. In this case we have Li-Yau's estimate

$$
|\nabla f|^{2}-f_{t} \leq \frac{n}{2 t}
$$

Let $U(t, Y)$ be the solution of the differential equation on the half line $(0, \infty)$ with a parameter $Y$

$$
\partial_{t} U+\frac{2}{n} U^{2}+2 K(Y+U)=0, \quad U(0)=\infty .
$$


If $Y<n K / 4$, then

$$
U(t, Y)=X(t, Y)-Y=-\frac{n K}{2}+\frac{n}{2 t} \frac{h(t, Y)}{2} \operatorname{cotanh} \frac{h(t, Y)}{2},
$$

with

$$
h(t, Y)=\frac{4 t}{n} \sqrt{n K} \sqrt{\frac{n K}{4}-Y} .
$$

If $Y>n K / 4$, then

$$
U(t, Y)=X(t, Y)-Y=-\frac{n K}{2}+\frac{n}{2 t} \frac{h(t, Y)}{2} \operatorname{cotan} \frac{h(t, Y)}{2}
$$

with

$$
h(t, Y)=\frac{4 t}{n} \sqrt{n K} \sqrt{Y-\frac{n K}{4}} .
$$

Therefore

$$
\lim _{Y \rightarrow n K / 4} U(t, Y)=\frac{n}{2 t}-\frac{n K}{2}
$$

and

$$
\lim _{Y \rightarrow n K / 4} \partial_{Y} U(t, Y)=-\frac{2 K t}{3} .
$$

Moreover, $U$ is a smooth function on $(0, \infty) \times \mathbb{R}$, and for any $t>0$, the function $Y \longrightarrow U(t, Y)$ is concave on $(-\infty, n K / 4)$. But it is not concave on $(n K / 4, \infty)$.

For any $Y_{0}<n K / 4$, we define a test function $G=t F, F=$ $|\nabla f|^{2}-f_{t}-B\left(t, f_{t}\right)$, where

$$
B(t, Y)=\partial_{Y} U\left(t, Y_{0}\right)\left(Y-Y_{0}\right)+U\left(t, Y_{0}\right) .
$$

Then by Bochner inequality, we get that

$$
\begin{aligned}
L G & \geq \frac{2}{n t} G^{2}+\left(\frac{4 B}{n}+2 K-\frac{1}{t}\right) G+t\left(\partial_{t} B+\frac{2}{n} B^{2}+2 K\left(f_{t}+B\right)\right) \\
& =\frac{2}{n t}\left(G+t \partial_{Y} U\left(t, Y_{0}\right)\left(f_{t}-Y_{0}\right)\right)^{2}+\left(\frac{4}{n} U\left(t, Y_{0}\right)+2 K-\frac{1}{t}\right) G .
\end{aligned}
$$

By the fact that

$$
\frac{4}{n} U\left(t, Y_{0}\right)+2 K-\frac{1}{t} \geq \frac{1}{t}, \quad \text { for all } Y_{0}<\frac{n K}{4}, t>0,
$$


we conclude by the maximum principle that $G \leq 0$. Therefore we have

Theorem 6. Let Ric $\geq K \geq 0$, and let $f=\log u$, where $u$ is a positive solution of the heat equation. Then

$$
|\nabla f|^{2}-f_{t} \leq \inf _{Y_{0}<n K / 4}\left(\partial_{Y} U\left(t, Y_{0}\right)\left(f_{t}-Y_{0}\right)+U\left(t, Y_{0}\right)\right),
$$

for any $t>0$. In particular, we have

$$
|\nabla f|^{2} \leq X\left(t, f_{t}\right), \quad \text { on } f_{t} \leq \frac{n K}{4},
$$

and

$$
|\nabla f|^{2}-f_{t} \leq \frac{2}{3} K t\left(\frac{n K}{4}-f_{t}\right)+\frac{n}{2 t}-\frac{n K}{2}
$$

Define a function $V(t, Y)$ by

$$
\begin{aligned}
& V(t, Y)=X(t, Y) \\
& =-\frac{n K}{2}+Y \\
& +\sqrt{n K} \sqrt{\frac{n K}{4}-Y} \operatorname{cotanh} \frac{2 t}{n} \sqrt{n K} \sqrt{\frac{n K}{4}-Y},
\end{aligned}
$$

when $Y<n K / 4$, and

$$
V(t, Y)=-\frac{n K}{2}+Y-\frac{2 K t}{3}\left(Y-\frac{n K}{4}\right),
$$

when $Y \geq n K / 4$. Then we can rewrite the estimates in Theorem 6 to be

$$
|\nabla f|^{2} \leq V\left(t, f_{t}\right), \quad \text { for all } t>0
$$

It is easily seen that there is a unique zero point of $V(t, Y)$ in $(-\infty, 0)$ for each $t>0$, denoted it by $Y_{1}(t)$. Then by the fact that $|\nabla f|^{2} \geq 0$, we have $f_{t} \geq Y_{1}(t)$.

If $n /(2 t)-n K / 4<0$, that is, if $t>2 / K$, then there is a unique zero point of $V(t, Y)$ in $(0, n K / 4]$, denoted by $Y_{2}(t)$, and again by the fact that $|\nabla f|^{2} \geq 0$, the estimate (67) yields that $f_{t} \leq Y_{2}(t)$. 
If $n /(2 t)-n K / 4>0$ and $-2 K t / 3+1<0$, that is, if $3 /(2 K)<$ $t<2 / K$, then we can see that the unique zero point of $V(t, Y)$ in $(0, \infty)$ is

$$
\frac{n K}{4}+\left(\frac{2 K t}{3}-1\right)^{-1}\left(\frac{n}{2 t}-\frac{n K}{4}\right)
$$

and by the same reasoning as the above, we have

$$
f_{t} \leq \frac{n K}{4}+\frac{1}{\frac{2 K t}{3}-1}\left(\frac{n}{2 t}-\frac{n K}{4}\right) .
$$

In these two cases, that is, if $t>3 /(2 K)$, there is a unique maximum value of $V(t, Y)$, attending at some point in $\left(Y_{1}(t), n K / 4\right]$, which is denoted by $V_{0}(t)$. Then by $(67)$, we have $|\nabla f|^{2} \leq V_{0}(t)$.

Thus we have proved the following

Theorem 7. Let Ric $\geq K \geq 0$, and let $f=\log u$, where $u$ is a positive solution of the heat equation. Then

$$
Y_{1}(t) \leq f_{t} \leq Y_{2}(t) \leq \frac{n K}{4}, \quad \text { for all } t>\frac{2}{K},
$$

(69) $Y_{1}(t) \leq f_{t} \leq \frac{n K}{4}+\frac{1}{\frac{2 K t}{3}-1}\left(\frac{n}{2 t}-\frac{n K}{4}\right)$, for all $t>\frac{3}{2 K}$,

and

$$
|\nabla f|^{2} \leq V_{0}(t), \quad \text { for all } t>\frac{3}{2 K}
$$

Now let us estimate $Y_{1}(t)$. To this end, let $\widetilde{U}$ be the solution of the differential equation

$$
\partial_{t} \widetilde{U}+\frac{2}{n} \widetilde{U}^{2}+2 \widetilde{K}(Y+\widetilde{U})=0, \quad \widetilde{U}(0)=\infty .
$$

Then $\widetilde{U}$ is given by the formula as for $U$ instead of $K$ by $\widetilde{K}$.

Let $W=t(U-\widetilde{U})$. Then for any $Y<n K / 4$, we have

$$
\begin{aligned}
-\partial_{t} W & =\frac{2}{n t} W^{2}+\left(\frac{4}{n} U+2 K-\frac{1}{t}\right) W+2(K-\widetilde{K})(Y+\widetilde{U}) t \\
& \geq \frac{2}{n t} W^{2}+\frac{1}{t} W+2(K-\widetilde{K})(Y+\widetilde{U}) t .
\end{aligned}
$$


In particular, if $K \geq \widetilde{K}=0$, then $\widetilde{U}=n /(2 t)$, and

$$
-\partial_{t} W \geq \frac{2}{n t} W^{2}+\frac{1}{t} W+2 K\left(Y+\frac{n}{2 t}\right) t .
$$

Applying the maximum principle to $W$ on $[0, t] \times \mathbb{R}$ for any $0<t \leq$ $(-n /(2 Y)) \vee 0$, we conclude that $W \leq 0$ for any $t \leq(-n /(2 Y) \vee 0$. Therefore we have proved the following

Proposition 1. If $K \geq 0$, then for any $Y, t>0$ such that $Y<n K / 4$, $Y+n /(2 t) \geq 0$, we have

$$
U(t, Y) \leq \frac{n}{2 t}
$$

As a consequence, we have

$$
Y_{1}(t) \geq-\frac{n}{2 t}, \quad \text { for all } t>0 .
$$

Our next goal is to bound $|\nabla f|^{2}-f_{t}$ for small time $t$.

Theorem 8. Let $\operatorname{Ric} \geq K>0$, and let $f=\log u$, where $u$ is a positive solution of the heat equation. Then for any $Y_{0}>n K / 4$, we have

$$
|\nabla f|^{2}-f_{t} \leq \partial_{Y} U\left(t, Y_{0}\right)\left(f_{t}-Y_{0}\right)+U\left(t, Y_{0}\right),
$$

for

$$
0<t \leq \frac{n \pi}{8 \sqrt{n K} \sqrt{Y_{0}-\frac{n K}{4}}} .
$$

Proof. Let $G=t F, F=|\nabla f|^{2}-f_{t}-B\left(t, f_{t}\right)$, where

$$
B(t, Y)=\partial_{Y} U\left(t, Y_{0}\right)\left(Y-Y_{0}\right)+U\left(t, Y_{0}\right) .
$$

Then by (63), we have

$$
L G \geq \frac{2}{n t}\left(G+t \partial_{Y} U\left(t, Y_{0}\right)\left(f_{t}-Y_{0}\right)\right)^{2}+\left(\frac{4}{n} U\left(t, Y_{0}\right)+2 K-\frac{1}{t}\right) G .
$$

However, when $Y_{0}>n K / 4$ and

$$
t \leq \frac{n \pi}{8 \sqrt{n K} \sqrt{Y_{0}-\frac{n K}{4}}},
$$


we have

$$
\begin{aligned}
\frac{4 t}{n} U(t, & \left.Y_{0}\right)+2 K t \\
& =h \operatorname{cotan} \frac{h}{2} \\
& =\frac{4 t}{n} \sqrt{n K} \sqrt{Y_{0}-\frac{n K}{4}} \operatorname{cotanh} \frac{2 t}{n} \sqrt{n K} \sqrt{Y_{0}-\frac{n K}{4}} \\
& \geq 2 \cos \frac{2 t}{n} \sqrt{n K} \sqrt{Y_{0}-\frac{n K}{4}} \\
& \geq 2 \cos \frac{\pi}{4} \\
& =\sqrt{2} .
\end{aligned}
$$

Therefore

$$
L G \geq \frac{\sqrt{2}-1}{t} G,
$$

and by the maximum principle, we get the conclusion.

Corollary 5. Let

$$
Y_{0}=\left(1+\frac{\pi^{2}}{64}\right) \frac{n K}{4} .
$$

Then for any $0<t \leq 2 / K$, we have

$$
|\nabla f|^{2}-f_{t} \leq U\left(t, f_{t}\right), \quad \text { on } f_{t} \leq Y_{0}
$$

and

$$
|\nabla f|^{2}-f_{t} \leq \partial_{Y} U\left(t, Y_{0}\right)\left(f_{t}-Y_{0}\right)+U\left(t, Y_{0}\right)
$$

Therefore, if

$$
E(t, Y)=X(t, Y)=Y+U(t, Y), \quad \text { if } Y \leq Y_{0},
$$

and

$$
E(t, Y)=\partial_{Y} U\left(t, Y_{0}\right)\left(Y-Y_{0}\right)+U\left(t, Y_{0}\right)+Y, \quad \text { if } Y>Y_{0},
$$


then

$$
|\nabla f|^{2} \leq E\left(t, f_{t}\right), \quad \text { for all } t \leq \frac{2}{K},
$$

where $Y_{0}$ is defined in Corollary 5 .

Putting all discussions together, we have the following

Theorem 9. Let $\operatorname{Ric} \geq K>0$, and let $f=\log u$, where $u$ is a positive solution of the heat equation. Then we have the following conclusions.

1) If $t \geq 2 / K$, then $|\nabla f|^{2} \leq X\left(t, f_{t}\right)$ and $-n /(2 t) \leq f_{t} \leq n K / 4$.

2) If $t \leq 2 / K$, then we have

$$
|\nabla f|^{2} \leq X\left(t, f_{t}\right), \quad \text { on } f_{t} \leq Y_{0},
$$

and

$$
|\nabla f|^{2}-f_{t} \leq \partial_{Y} U\left(t, Y_{0}\right)\left(f_{t}-Y_{0}\right)+U\left(t, Y_{0}\right), \quad \text { on } f_{t}>Y_{0},
$$

where

$$
Y_{0}=\left(1+\frac{\pi^{2}}{64}\right) \frac{n K}{4}
$$

\section{Harnack inequalities.}

In this section we first show how to deduce a Harnack inequality from a gradient estimate, although it is very standard, see [9]. Then we prove the main Harnack estimates.

The link between Harnack inequalities and gradient estimates is given in the following

Proposition 2. Let $M$ be a complete Riemannian manifold, and let $f=\log u$; where $u$ is a positive solution of the heat equation. Suppose that

$$
|\nabla f|^{2} \leq \psi\left(t, f_{t}\right), \quad \text { for all } t>0
$$

where $\psi:(0, \infty) \times \mathbb{R} \longrightarrow \mathbb{R}$ is a continuous function, then

$$
\frac{u(t, x)}{u(t+s, y)} \leq \exp \left(\int_{t}^{t+s} K\left(\sigma, \frac{\rho}{s}\right) d \sigma\right),
$$


where $\rho$ is the geodesic distance between $x$ and $y$, and

$$
K(t, \alpha)=\sup _{\{Y: \psi(t, Y) \geq 0\}}(\alpha \sqrt{\psi(t, Y)}-Y) .
$$

PROOF. The proof of this proposition is straightforward. Let $\gamma$ be a minimal geodesic joining $x$ and $y$, so that $\gamma(0)=y$ and $\gamma(1)=x$. If $\rho=d(x, y)$, then $|\dot{\gamma}|=\rho$. Define

$$
p(\sigma)=\left(\gamma(\sigma),(1-\sigma) t_{2}+t_{1} \sigma\right), \quad t_{2}=t+s, t_{1}=t .
$$

Then $p(0)=\left(y, t_{2}\right)$ and $p(1)=\left(x, t_{1}\right)$. Set $\eta(\sigma)=f(p(\sigma))$. It is clear that

$f\left(t_{1}, x\right)-f\left(t_{2}, y\right)=\eta(1)-\eta(0)=\int_{0}^{1} \dot{\eta}(\sigma) d \sigma=\int_{0}^{1}\left(\langle\nabla f, \dot{\gamma}\rangle-s f_{t}\right) d \sigma$

with $t=(1-\sigma) t_{2}+t_{1} \sigma$. We end up with

$$
\begin{aligned}
f\left(t_{1}, x\right)-f\left(t_{2}, y\right) & \leq \int_{0}^{1}\left(\rho|\nabla f|-s f_{t}\right) d \sigma \\
& \leq \int_{0}^{s}\left(\frac{\rho}{s}|\nabla f|-f_{t}\right) d \sigma \\
& \leq \int_{0}^{s} K\left(\sigma, \frac{\rho}{s}\right) d \sigma .
\end{aligned}
$$

From this result and the previous gradient estimates, we may now prove Harnack inequalities: we shall first establish the simplest one, for which the computations are easy: it follows from the gradient estimate (53).

Theorem 10. Let Ric $\geq-K, K \geq 0$, and let $u$ be a positive solution of the heat equation. Then

$\frac{u(t, x)}{u(t+s, y)} \leq\left(\frac{t+s}{t}\right)^{n / 2}$

$$
\cdot \exp \left(\frac{(\rho+\sqrt{n K} s)^{2}}{4 s}+\frac{\sqrt{n K}}{2} \min \left\{(\sqrt{2}-1) \rho, \frac{\sqrt{n K}}{2} s\right\}\right),
$$

for all $s>0, t \geq 0, x, y \in M$, where $\rho=d(x, y)$. 
Proof. Let $f=\log u$, and $\alpha>0$ be fixed.

If $f_{t}>-n K / 4$, then the gradient estimate (53) yields that

$$
|\nabla f|^{2} \leq\left(\gamma+\sqrt{\frac{n K}{4}}\right)^{2}+\frac{n}{2 t}
$$

where $\gamma=\sqrt{f_{t}+n K / 4}$, so that

$$
-f_{t}=\frac{n K}{4}-\gamma^{2}
$$

Denote by

$$
u=\sqrt{\left(\gamma+\sqrt{\frac{n K}{4}}\right)^{2}+\frac{n}{2 t}} \geq 0 .
$$

Then $|\nabla f| \leq u$ and

$$
\begin{aligned}
-f_{t} & =\frac{n K}{4}-\left(\sqrt{u^{2}-\frac{n}{2 t}}-\sqrt{\frac{n K}{4}}\right)^{2} \\
& =-u^{2}+\frac{n}{2 t}+\sqrt{n K} \sqrt{u^{2}-\frac{n}{2 t}} \\
& \leq-u^{2}+\frac{n}{2 t}+\sqrt{n K} u .
\end{aligned}
$$

Hence in this case we have

$$
\alpha|\nabla f|-f_{t} \leq(\alpha+\sqrt{n K}) u-u^{2}+\frac{n}{2 t} \leq \frac{(\alpha+\sqrt{n K})^{2}}{4}+\frac{n}{2 t}
$$

If $f_{t} \leq-n K / 4$, then on one hand the estimate (54) implies that

$$
-f_{t} \leq-|\nabla f|^{2}+\frac{n}{2 t}+\frac{n K}{2}
$$

so that

$$
\begin{aligned}
\alpha|\nabla f|-f_{t} & \leq \frac{\alpha^{2}}{4}+\frac{n}{2 t}+\frac{n K}{2} \\
& =\frac{(\alpha+\sqrt{n K})^{2}}{4}+\frac{n}{2 t}+\frac{n K}{4}-\frac{\sqrt{n K}}{2} \alpha \sigma .
\end{aligned}
$$


On other hand, by the estimate (54), we have

Therefore

$$
|\nabla f|^{2}-f_{t} \leq \frac{2 K t}{3}\left(f_{t}+\frac{n K}{4}\right)+\frac{n}{2 t}+\frac{n K}{2} .
$$

$$
\begin{aligned}
& \alpha|\nabla f|-f_{t} \leq|\alpha \nabla f|-\frac{1}{1+\frac{2 K}{3} t}|\nabla f|^{2} \\
& +\frac{1}{1+\frac{2 K}{3} t}\left(\frac{n}{2 t}+\frac{n K}{4}\right)+\frac{n K}{4} \\
& \leq \frac{\alpha^{2}}{4}+\left(1-\frac{1}{\sqrt{1+\frac{2 K}{3} t}}\right) \alpha|\nabla f| \\
& +\frac{1}{1+\frac{2 K}{3} t}\left(\frac{n}{2 t}+\frac{n K}{4}\right)+\frac{n K}{4} \\
& \leq \frac{\alpha^{2}}{4}+\left(1-\frac{1}{\sqrt{1+\frac{2 K}{3} t}}\right) \sqrt{\frac{n}{2 t}+\frac{n K}{2}} \alpha \\
& +\frac{n}{2 t}+\frac{n K}{4}-\frac{n K}{12} \frac{1}{1+\frac{2 K}{3} t} \\
& \leq \frac{\alpha^{2}}{4}+\frac{\frac{2 K}{3} t \sqrt{\frac{n}{2 t}+\frac{n K}{2}}}{\sqrt{1+\frac{2 K}{3} t}\left(1+\sqrt{1+\frac{2 K}{3} t}\right)} \alpha+\frac{n}{2 t}+\frac{n K}{4} \\
& \leq \frac{\alpha^{2}}{4}+\sqrt{\frac{n K}{2}} \alpha+\frac{n}{2 t}+\frac{n K}{4} \\
& =\frac{(\alpha+\sqrt{n K})^{2}}{4}+\frac{n}{2 t}+\frac{\sqrt{2}-1}{2} \sqrt{n K} \alpha .
\end{aligned}
$$

Hence, if $f_{t}+n K / 4<0$, then we have

$$
\begin{aligned}
\alpha|\nabla f|-f_{t} \leq & \frac{(\alpha+\sqrt{n K})^{2}}{4}+\frac{n}{2 t} \\
& +\frac{\sqrt{n K}}{2} \min \left\{(\sqrt{2}-1) \alpha, \frac{\sqrt{n K}}{2}\right\} .
\end{aligned}
$$


Therefore, by (79) and (81), we always have the following estimate

$$
\begin{aligned}
\alpha|\nabla f|-f_{t} \leq & \frac{(\alpha+\sqrt{n K})^{2}}{4}+\frac{n}{2 t} \\
& +\frac{\sqrt{n K}}{2} \min \left\{(\sqrt{2}-1) \alpha, \frac{\sqrt{n K}}{2}\right\},
\end{aligned}
$$

for any $\alpha \geq 0$.

Using now the previous proposition, we get

$$
\begin{aligned}
& f\left(t_{1}, x\right)-f\left(t_{2}, y\right) \\
\leq & \int_{0}^{1}\left(\frac{(\rho+\sqrt{n K} s)^{2}}{4 s}+\frac{n}{2 t} s+\frac{\sqrt{n K}}{2} \min \left\{(\sqrt{2}-1) \rho, \frac{\sqrt{n K}}{2} s\right\}\right) d \sigma,
\end{aligned}
$$

which yields the Harnack inequality.

Remark. As we pointed out in the introduction, S. T. Yau mentioned to us that he obtained a similar Harnack inequality.

Now we turn to prove the main Harnack estimate. Let Ric $\geq-K$ for some constant $K \geq 0$. We have seen that the main point is to estimate $\alpha|\nabla f|-f_{t}$ for $\alpha>0$.

For any $Y_{0}>-n K / 4$, we have

$$
|\nabla f|^{2}-f_{t} \leq C\left(t, Y_{0}\right)+\partial_{Y} C\left(t, Y_{0}\right)\left(f_{t}-Y_{0}\right),
$$

and therefore

$$
-f_{t} \leq-\frac{|\nabla f|^{2}}{1+\partial_{Y} C\left(t, Y_{0}\right)}+\frac{C\left(t, Y_{0}\right)-Y_{0} \partial_{Y} C\left(t, Y_{0}\right)}{1+\partial_{Y} C\left(t, Y_{0}\right)} .
$$

Hence for any $\alpha>0$, we have

$$
\begin{aligned}
\alpha|\nabla f|-f_{t} \leq & -\frac{1}{1+\partial_{Y} C\left(t, Y_{0}\right)}|\nabla f|^{2}+\alpha|\nabla f|+\frac{C\left(t, Y_{0}\right)-Y_{0} C\left(t, Y_{0}\right)}{1+\partial_{Y} C\left(t, Y_{0}\right)} \\
\leq & \frac{\alpha^{2}}{4}\left(1+\partial_{Y} C\left(t, Y_{0}\right)\right)+\frac{C\left(t, Y_{0}\right)-Y_{0} \partial_{Y} C\left(t, Y_{0}\right)}{1+\partial_{Y} C\left(t, Y_{0}\right)} \\
(83) \quad & \frac{\alpha^{2}}{4}+\left(\partial_{Y} C\left(t, Y_{0}\right)\left(\frac{\alpha^{2}}{4}-Y_{0}\right)+C\left(t, Y_{0}\right)\right) \\
& -\frac{\partial_{Y} C\left(t, Y_{0}\right)}{1+\partial_{Y} C\left(t, Y_{0}\right)}\left(C\left(t, Y_{0}\right)+\partial_{Y} C\left(t, Y_{0}\right)\left(0-Y_{0}\right)\right) .
\end{aligned}
$$


Letting $Y_{0}=\alpha / 4$ in (83), we get that

$$
\alpha|\nabla f|-f_{t} \leq \frac{\alpha^{2}}{4}+C\left(t, \frac{\alpha^{2}}{4}\right)-A\left(t, \frac{\alpha^{2}}{4}\right)
$$

where

$$
A(t, Y)=\frac{\partial_{Y} C(t, Y)\left(C(t, Y)-Y \partial_{Y} C(t, Y)\right)}{1+\partial_{Y} C(t, Y)}
$$

Notice that $A(t, Y) \geq 0$ when $Y \geq 0$. Therefore we have proved the following

Theorem 11. Let Ric $\geq-K ; K \geq 0$, and let $u$ be a positive solution of the heat equation. Then

(85) $\frac{u(t, x)}{u(t+s, y)} \leq \exp \left(\frac{\rho^{2}}{4 s}+\int_{t}^{t+s}\left(C\left(\sigma, \frac{\rho^{2}}{4 s^{2}}\right)-A\left(\sigma, \frac{\rho^{2}}{4 s^{2}}\right)\right) d \sigma\right)$,

for any $t \geq 0, s>0$ and $x, y \in M$, where $\rho$ is the geodesic distance between $x$ and $y$.

REMARK. Although we have the simple fact that

$$
C(t, Y) \leq \sqrt{n K} \sqrt{Y+\frac{n K}{4}}+\frac{n}{2 t}+\frac{n K}{2},
$$

for any $Y>-n K / 4$, however, unlike $C(t, Y)$ whose linearization at any point $Y \geq-n K / 4$ is an upper bound of $|\nabla f|^{2}-f_{t}$, the linearization of

$$
\sqrt{n K} \sqrt{Y+\frac{n K}{4}}+\frac{n K}{2}+\frac{n}{2 t}
$$

at some points may not be an upper bound of $|\nabla f|^{2}-f_{t}$. In this sense, therefore, the analysis via $C(t, Y)$ is even simpler and yields much stronger conclusions. This is also the reason why we give an independent proof of Theorem 10. 
Since

$$
\begin{aligned}
& C(t, Y)=\frac{n K}{2}+ \frac{n}{2 t} \frac{b(t, Y)}{2} \operatorname{cotanh} \frac{b(t, Y)}{2} \\
&=\frac{n K}{2}+\sqrt{n K} \sqrt{Y+\frac{n K}{4}} \operatorname{cotanh}\left(\frac{2 t}{n} \sqrt{n K} \sqrt{Y+\frac{n K}{4}}\right) \\
& \int_{t}^{t+s} C(\sigma, Y) d \sigma=\frac{n K}{2} s+\frac{n}{2} \int_{(2 / n) t \sqrt{n K} \sqrt{Y+n K / 4}}^{(2 / n)(t+s) \sqrt{n K} \sqrt{Y+n K / 4}} \operatorname{cotanh} \sigma d \sigma \\
&=\frac{n K}{2} s+\frac{n}{2} \log \left(\frac{\sinh \frac{2}{n}(t+s) \sqrt{n K} \sqrt{Y+\frac{n K}{4}}}{\sinh \frac{2}{n} t \sqrt{n K} \sqrt{Y+\frac{n K}{4}}}\right)
\end{aligned}
$$

so that we have the following

Corollary 6. Let Ric $\geq-K ; K \geq 0$, and let $u$ be a positive solution of the heat equation. Then

$$
\begin{aligned}
& \frac{u(t, x)}{u(t+s, y)} \\
& \quad \leq\left(\frac{\sinh \left(\frac{2}{n}(t+s) \sqrt{n K} \sqrt{\frac{\rho^{2}}{4 s^{2}}+\frac{n K}{4}}\right)}{\sinh \left(\frac{2}{n} t \sqrt{n K} \sqrt{\frac{\rho^{2}}{4 s^{2}}+\frac{n K}{4}}\right)}\right)^{n / 2} E(\rho, s, t),
\end{aligned}
$$

where

$$
E(\rho, s, t)=\exp \left(\frac{\rho^{2}}{4 s}+\frac{n K}{2} s-\int_{t}^{t+s} A\left(\sigma, \frac{\rho^{2}}{4 s^{2}}\right) d \sigma\right) .
$$

Applying Corollary 6 to the heat kernel we have

$$
H(t, x, y) \geq\left(\frac{\frac{2}{n} \sqrt{n K} \sqrt{\frac{\rho^{2}}{4 t^{2}}+\frac{n K}{4}}}{4 \pi \sinh \left(\frac{2 t}{n} \sqrt{n K} \sqrt{\frac{\rho^{2}}{4 t^{2}}+\frac{n K}{4}}\right)}\right)^{n / 2} E(\rho, t, 0)^{-1}
$$

$$
=\frac{1}{(4 \pi t)^{n / 2}}\left(\frac{\sqrt{\frac{K}{n}} \sqrt{\rho^{2}+n K t^{2}}}{\sinh \left(\sqrt{\frac{K}{n}} \sqrt{\rho^{2}+n K t^{2}}\right)}\right)^{n / 2} E(\rho, t, 0)^{-1},
$$


where

$$
E(\rho, t, 0)^{-1}=\exp \left(-\frac{\rho^{2}}{4 t}-\frac{n K}{2} t+\int_{0}^{t} A\left(\sigma, \frac{\rho^{2}}{4 t^{2}}\right) d \sigma\right) .
$$

Remark. Proposition 2 together with the gradient estimates for the positive Ricci curvature manifolds yields a Harnack inequality. However, its form is quite complicated. Since the upper bound function $\psi(t, Y)$ is in general nonlinear, we can improve the Harnack inequality in Proposition 2 by varying the time speed, that is, replacing the straight line joining $t$ and $t+s$ by a curve. Therefore we decided to write down the explicit Harnack inequalities for positive Ricci curvature manifolds together with the compact manifold case in a separate paper.

\section{Extensions.}

The same arguments in previous sections can be applied to the case when the manifold $M$ with convex boundary $\partial M$; the second fundamental form $\pi$ of the boundary $\partial M$ is nonnegative. This is because of the fact that if $\partial u / \partial \nu=0$ on the boundary; where $\nu$ denotes the pointed out normal vector field; then

$$
\frac{\partial|\nabla u|^{2}}{\partial \nu}=-2 \pi(\nabla u, \nabla u)
$$

so that we can use the Hopf maximum principle when $\pi \geq 0$. We only write down a theorem in this case.

Theorem 12. Let $M$ be a complete Riemannian manifold with a convex boundary $\partial M$, and let $u$ be a positive solution of the heat equation

$$
\begin{gathered}
\left(\Delta-\partial_{t}\right) u=0, \quad \text { on }[0, \infty) \times M, \\
\partial_{\nu} u=0, \quad \text { on }(0, \infty) \times \partial M .
\end{gathered}
$$

Let $f=\log u$. Then

$$
|\nabla f|^{2}-f_{t} \leq C\left(t, f_{t}\right), \quad \text { on } f_{t} \geq-\frac{n K}{4}
$$

and

$$
|\nabla f|^{2}-f_{t} \leq \partial_{Y} C\left(t, Y_{0}\right)\left(f_{t}-Y_{0}\right)+C\left(t, Y_{0}\right)
$$


for all $t>0, Y_{0} \geq-n K / 4$.

There is a further generalisation of our gradient estimates and Harnack inequalities to a general elliptic operator. We only state a result.

Let $M$ be a complete manifold (without or with a convex boundary), and let $\Delta_{B}=\Delta+B$ be an elliptic operator; where $B$ is a $C^{2}$-vector field. Assume that $\Delta_{B}$ satisfies a curvature-dimension inequality (see $[1])$

$$
\Gamma_{2}(g, g) \geq \frac{1}{m}\left(\Delta_{B} g\right)^{2}-K \Gamma(g, g), \quad \text { for all } g \in C^{\infty}(M),
$$

for some constants $m>0$ and $K \geq 0$, where by definition $\Gamma(f, g)=$ $\langle\nabla f, \nabla g\rangle$, and

$$
\Gamma_{2}(f, g)=\frac{1}{2}\left(\Delta_{B}(f g)-\Gamma\left(\Delta_{B} f, g\right)-\Gamma\left(f, \Delta_{B} g\right)\right) .
$$

This condition is satisfied if and only if

$$
\text { Ric }-\nabla_{B}^{s}-\frac{1}{m-n} B \otimes B \geq-K,
$$

where $m \geq n, n=\operatorname{dim} M$, Ric denotes the Ricci curvature and

$$
\nabla_{B}^{s}(\xi, \eta)=\frac{1}{2}\left(\left\langle\nabla_{\xi} B, \eta\right\rangle+\left\langle\nabla_{\eta} B, \xi\right\rangle\right), \quad \text { for all } \xi, \eta \in T M .
$$

If $f=\log u, u$ is a positive solution of the heat equation

$$
\left(\Delta_{B}-\partial_{t}\right) u=0, \quad \text { on }[0, \infty) \times M,
$$

(in the case that the boundary $\partial M \neq 0$, we further assume that $u$ satisfies the Neumann boundary condition), then

$$
|\nabla f|^{2}-f_{t} \leq C\left(t, f_{t}\right), \quad \text { on } f_{t} \geq-\frac{m K}{4}
$$

and

$$
|\nabla f|^{2}-f_{t} \leq \partial_{Y} C\left(t, Y_{0}\right)\left(f_{t}-Y_{0}\right)+C\left(t, Y_{0}\right), \quad \text { for all } Y_{0} \geq-\frac{m K}{4}
$$

where $C$ is the solution of the differential equation

$$
\partial_{t} C+\frac{2}{m} C^{2}-2 K(Y+C)=0, \quad C(0)=\infty .
$$




\section{Appendix.}

The goal of this appendix is to give a proof of Yau's estimate (6). We will use the same notations as in Section 2.

Let $F=|\nabla f|^{2}-f_{t}-Q\left(t,|\nabla f|^{2}\right)$ for some positive function $Q$ which will be given later, and $G=t F$.

It is easily seen that

$$
\begin{aligned}
L F & =\left(1-\partial_{X} Q\right) L|\nabla f|^{2}+\partial_{t} Q-\left.\left.\partial_{X}^{2} Q|\nabla| \nabla f\right|^{2}\right|^{2} \\
& =2\left(1-\partial_{X} Q\right) \Gamma_{2}(f, f)-\left.\left.\partial_{X}^{2} Q|\nabla| \nabla f\right|^{2}\right|^{2}+\partial_{t} Q,
\end{aligned}
$$

and therefore if $\partial_{X}^{2} Q \leq 0,1-\partial_{X} Q \geq 0$, then we have

$$
\begin{aligned}
L G & =-F+2 t\left(1-\partial_{X} Q\right) \Gamma_{2}(f, f)-\left.\left.t \partial_{X}^{2} Q|\nabla| \nabla f\right|^{2}\right|^{2}+t \partial_{t} Q \\
& \geq-F+2 t\left(1-\partial_{X} Q\right)\left(\frac{1}{n}\left(|\nabla f|^{2}-f_{t}\right)^{2}-K|\nabla f|^{2}\right)+t \partial_{t} Q .
\end{aligned}
$$

However $|\nabla f|^{2}-f_{t}=F+Q$, so that

$$
\begin{aligned}
L G \geq & -F+2 t\left(1-\partial_{X} Q\right)\left(\frac{1}{n}(F+Q)^{2}-K|\nabla f|^{2}\right)+t \partial_{t} Q \\
= & 2 t\left(1-\partial_{X} Q\right) \frac{F^{2}}{n}+\left(-1+2 t\left(1-\partial_{X} Q\right) \frac{2 Q}{n}\right) F \\
& +2 t\left(1-\partial_{X} Q\right)\left(\frac{Q^{2}}{n}-K|\nabla f|^{2}\right)+t \partial_{t} Q .
\end{aligned}
$$

Thus, if

$$
\partial_{X}^{2} Q \leq 0, \quad \lim _{t \rightarrow 0} t Q(t, X) \leq 0,
$$

$$
\frac{4 t Q}{n}\left(1-\partial_{X} Q\right) \geq 1, \quad 1-\partial_{X} Q>0
$$

and

$$
\partial_{t} Q+2\left(1-\partial_{X} Q\right)\left(\frac{Q^{2}}{n}-K X\right) \geq 0
$$

for all $t>0, X \geq 0$, then, if the manifold is compact,

$$
|\nabla f|^{2}-f_{t} \leq Q\left(t,|\nabla f|^{2}\right), \quad \text { for all } t>0 .
$$


Theorem 13. Let Ric $\geq-K$ for some non-negative constant $K$, and let $u$ be a positive solution of the heat equation and $f=\log u$. Then

$$
|\nabla f|^{2}-f_{t} \leq \sqrt{n K} \sqrt{|\nabla f|^{2}+\frac{n}{2 t}+\frac{n K}{4}}+\frac{n}{2 t} .
$$

Proof. For simplicity, let $a=n /(2 t)+n K / 4$. Then

$$
Q(t, X)=m \sqrt{X+a}+\frac{n}{2 t}, \quad m=\sqrt{n K} .
$$

Therefore

$$
\partial_{X}^{2} Q=-\frac{m}{4(X+a) \sqrt{X+a}} \leq 0
$$

and

$$
\begin{aligned}
\partial_{t} Q+2( & \left.-\partial_{X} Q\right)\left(\frac{Q^{2}}{n}-K X\right) \\
& =\frac{2 m \sqrt{X+a}}{t}-\frac{m^{2}}{t}-\frac{n m}{4 t^{2} \sqrt{X+a}}+2\left(1-\frac{m}{2 \sqrt{X+a}}\right) \frac{a}{n} \\
& \geq \frac{m}{t}\left((2 \sqrt{a}-m)-\frac{n}{4 t \sqrt{a}}\right) \\
& =\frac{m}{t}\left(\frac{4 a-m^{2}}{2 \sqrt{a}+m}-\frac{n}{4 t \sqrt{a}}\right) \\
& =\frac{m}{t}\left(\frac{2 n}{t(2 \sqrt{a}+m)}-\frac{n}{4 \sqrt{a} t}\right) \\
& \geq \frac{m}{t}\left(\frac{2 n}{t(4 \sqrt{a})}-\frac{n}{4 \sqrt{a} t}\right) \\
& >0
\end{aligned}
$$

Thus condition (91) is satisfied.

Let us now check the condition (90). It is easily seen that

$$
\begin{aligned}
\frac{4 Q}{n}\left(1-\partial_{X} Q\right) & =\frac{4 m}{n}\left(\sqrt{X+a}-\frac{m}{2}\right)+\frac{1}{t}\left(2-\frac{m}{\sqrt{X+a}}\right) \\
& \geq \frac{4 m}{n}\left(\sqrt{\frac{n}{2 t}+\frac{n K}{4}}-\frac{\sqrt{n K}}{2}\right)+\frac{1}{t}\left(2-\frac{m}{\sqrt{\frac{n}{2 t}+\frac{n K}{4}}}\right)
\end{aligned}
$$


Therefore, when $n /(2 t) \geq 3 n K / 4$,

$$
2-\frac{m}{\sqrt{\frac{n}{2 t}+\frac{n K}{4}}} \geq 1
$$

so that

$$
\frac{4 Q}{n}\left(1-\partial_{X} Q\right) \geq \frac{1}{t}
$$

and when $n /(2 t) \leq 3 n K / 4$,

$$
\frac{4 Q}{n}\left(1-\partial_{X} Q\right) \geq \frac{2 m}{t} \frac{1}{\sqrt{\frac{n}{2 t}+\frac{n K}{4}+\frac{\sqrt{n K}}{2}}} \geq \frac{4}{3 t}>\frac{1}{t} .
$$

Hence condition (90) is satisfied. Therefore we proved Theorem 13 for compact manifolds. If the manifold is non-compact, we may use the generalised maximum principle to go through the proof.

Acknowledgements. The authors thank Professor S. T. Yau for his comments on their paper [2]. The second author was supported by a grant from the Ministry of Foreign Affairs, France.

\section{References.}

[1] Bakry, D., Emery, M., Diffusions hypercontractives. Sém. de Probab. XIX. Lecture Notes in Math. 1123, 177-206, 1985.

[2] Bakry, D., Qian, Z., Gradient estimates and Harnack inequality on a complete manifold. Preprint, 1996.

[3] Bakry, D., Qian, Z., On Harnack estimates for positive solutions of the heat equation on a complete manifold. C. R. Acad. Sci. Paris $\mathbf{3 2 4}$ (1997), 1037-1042.

[4] Cheeger, J., Yau,S.-T., A lower bound for the heat kernel. Comm. Pure Appl. Math. 34 (1981), 465-480.

[5] Cheng, S. Y., Yau, S.-T., Differential equations on Riemannian manifolds and their geometric applications. Comm. Pure Appl. Math. 28 (1975), 333-354.

[6] Davies, E. B., Heat Kernels and Spectral Theory. Cambridge Tracts in Math. Cambridge Univ. Press 92, 1989. 
[7] Gilbarg, D., Trudinger, N. S., Elliptic Partial Differential Equations of Second Order. Springer-Verlag, 1977.

[8] Hamilton, R. S., A matrix Harnack estimate for the heat equation. Comm. in Analysis and Geometry. 1 (1993), 113-126.

[9] Li, P., Yau S.-T., On the parabolic kernel of the Schrödinger operator. Acta Math. 156 (1986), 153-201.

[10] Schoen, R., Yau, S.-T., Lectures on Differential Geometry. International Press, 1995.

[11] Sturm, K.-T., Heat kernel bounds on manifolds. Math. Ann. 292 (1992), 149-162.

[12] Yau, S.-T., Harmonic functions on complete Riemannian manifolds. Comm. Pure Appl. Math. 28 (1975), 201-228.

[13] Yau, S.-T., Some function-theoretic properties of complete Riemannian manifolds and their applications to geometry. Indiana Univ. Math. J. 25 (1976), 659-670.

[14] Yau, S.-T., On the Harnack inequalities of partial differential equations. Comm. in Analysis and Geometry 2 (1994), 431-450.

[15] Yau, S.-T., Harnack inequality for non-self-adjoint evolution equations. Math. Research Lett. 2 (1995), 387-399.

Recibido: 26 de noviembre de 1.997

Dominique Bakry

Laboratoire de Statistique et Probabilites

CNRS

Université Paul Sabatier 118, route de Narbonne

31062 Toulouse Cedex, FRANCE

bakry@cict.fr

and

Zhongmin M. Qian

Department of Mathematics

Imperial College of Science, Technology and Medicine

180 Queen's Gate

London SW7 2BZ, UNITED KINGDOM

and

Shanghai TieDao University, CHINA

z.qian@ic.ac.uk 